\title{
ESPÈCES NOUVELLES
}

\section{Acariens}

Ixodes pacificus, R. A. Cooley et G. M. Kohls. Ixodidé. Colombie Britannique et Californie. Pan-Pacific Ent., XIX, 1943, 4, 139-147.

Ixodes conepati R. A. Cooley et G. M. Kohls. Ixodidé. Conepatus. Texas. Pan-Pacific Ent., XIX, 1943, 4, 139-147.

Ornithodorus dugesi L. Mazzotti. Ixodidé. Mexique. Rev. Inst. Salub. Enferm. trop., IV, 1943, 4, 371-374.

Ornithodorus nereensis E. N. Pavlovsky. Karakala, Turkmenistan. C.R. Acad. Sci. U.R.S.S., XXXI, 1941, 4, 408.

Amblyomma avecolens R. A. Cooley et G. M. Kohls. Ixodidé. Vireo griseus (Oiseau). Sorita, Kennedy, Texas. J. Parasit., N.-Y., XXX, 1944, 77.

Ornithodorus aquilae R. A. Cooley. Ixodidé. Oiseaux. Wyoming et Colorado. J. Parasit., XXX, 1944, 5, 287.

Ixodes ozarkus R. A. Cooley. Ixodidé. Daims et chiens. Arkansas. J. Parasit., XXX, 1944, 5, 287.

Microtrombidium maculatum C. D. Michender. Thrombididé. Moustiques. Panama. Ann. ent. Soc. Amer., XXXIX, 1946, 3, 349.

Microtrombidium pistiae C. D. Michender. Thrombididé. Chironomes (morts). Panama. Ann. ent. Soc. Amer., XXXIX, 1946, 3, 349.

Ixodes montoyanus R. A. Cooley. Ixodidé. Nasuella olivacea. Monserrate, Bogota, Colombie. Bol. Ofic. Sanit. Panamer. Wash., XXIII, 1944, 12 , 1090 .

Ixodes neotomae R. A. Cooley. Ixodidé. Neotoma microtis. Los Angeles, Californie. Pan-Pacific Ent., XX, 1944, 1, 7.

Ixodes ozarkus R. A. Cooley. Ixodidé. Chien. Huntsville, Arkansas. J. Parasit., XXX, 1944, 5, 287.

Ixodes Iongiscutatum J. J. Boero. Ixodidé. Rev. Assoc. Med. Argent., LVIII, 1944, 534, 353.

Ixodes peromysci G. F. Augustson. Ixodidé. Peromyscus maniculatus elusus. Californie. Bull. S. Calif. Acad. Sci., XXXVIII, 1940, 3, 191.

Ornithodorus dyeri R. A. Cooley et G. M. Kohls. Ixodidé. Pul. Health Rep. Washingston, LV, 1940, 21, 925.

Otobius Iagophilus R. A. Cooley et G. M. Kohls. Ixodidé. Lapin. Publ. Health Rep. Washington, LV, 1940, 21, 928.

Le Gérant: Georges Masson.

Masson ET Cae Editeurs, Paris

Dépôt légal : 1951 (1 $1^{\text {er }}$ trimestre). - Numéro d'ordre : 1.298

à Cahors (France). - 81.187 . - C.O.L. 31.2330

Imprimé par Imp A. CouesLant (personnel intéressé) 